\title{
Equação de Hazen-Williams corrigida para água residuária proveniente da suinocultura
}

$\overline{\text { Silvio C. Sampaio }{ }^{1} \text {, Kátia Cilene' }{ }^{2} \text {, Márcio A. Vilas Boas }{ }^{1} \text {, Manoel M. F. de Queiroz }{ }^{1} \text {, Benedito M. Gomes }{ }^{1} \text { \& Ajadir Fazolo }}{ }^{1}$

\section{RESUMO}

O objetivo do trabalho foi avaliar a perda de carga em tubulações comerciais de aço zincado, ferro galvanizado e PVC, com diferentes diâmetros, com o intuito de determinar fatores de correção para a equação de Hazen-Williams em função da concentração de sólidos totais, em águas residuárias oriundas da suinocultura. Os resultados mostraram que a perda de carga possui comportamento linear em função da concentração de sólidos totais da água residuária proveniente da suinocultura; as perdas de carga determinadas a partir dos fatores de correção parcial e global, apresentaram superestimativa e subestimativa média de 0,7 e $13 \%$, respectivamente.

Palavras-chave: reúso de água, irrigação, hidráulica

\section{Hazen-Williams equation corrected for swine wastewater}

\begin{abstract}
The objective of this study was to evaluate the head losses in commercial tubes of zinc, galvanized iron and PVC, with different diameters, aiming to determinate correction factors for Hazen-Willams equation as a function of total solids in swine wastewater. The results showed that the head loss has a linear relation with the total solids of swine wastewater; the head losses estimated by partial and global correction factors showed mean overestimate and underestimate of 0.7 and $13 \%$, respectively.
\end{abstract}

Key words: water reuse, irrigation, hydraulics

${ }^{1}$ RHESA/UNIOESTE. Rua Universitária, 2069. CEP 85819-110, Cascavel, PR. Fone: (45) 3220-3134. E-mail: ssampaio@unioeste.br; vilasma@unioeste.br; mfqueiroz@unioeste.br; bmgomes@unioeste.br; afazolo@unioeste.br

${ }^{2}$ RHESA/UNIOESTE. Discente do curso de Engenharia Agrícola. Fone: (45) 3220-3153. E-mail: cilene@unioeste.br 


\section{INTRODUÇÃO}

A irrigação é uma técnica que tinha, antigamente, o único objetivo de fornecer a água necessária ao desenvolvimento das plantas mas, com o passar do tempo e com o avanço da tecnologia, foi possível atribuir-lhe novas finalidades, como: proporcionar condições favoráveis de umidade para preparo do solo, plantio, combate a geadas, aplicação de defensivos, fertilizantes e resíduos industriais, aplicação de produtos químicos possibilitando o controle da salinidade do solo associada à drenagem agrícola e, também, a utilização de águas residuárias provenientes de estábulos e pocilgas na irrigação.

No caso da utilização de água residuárias, recomenda-se o uso da irrigação pressurizada, por permitir maior controle na aplicação, destacando-se a aspersão convencional, autopropelido, sistema linear móvel e pivô central, o gotejamento e a microaspersão.

Existe forte tendência de que a utilização de águas residuárias na fertirrigação seja cada vez maior, até por consistir um fator econômico fundamental para o irrigante, haja vista que a água captada em rios e lagos passará a ser taxada de acordo com a nova legislação sobre o uso da água, em todos os estados brasileiros mas há, também a necessidade extrema de se desenvolver estudos que caracterizem o comportamento hidráulico dessas águas residuárias quando submetidas ao escoamento em condutos forçados, cujo comportamento pode ser caracterizado através da determinação de alguns parâmetros, como a viscosidade ou a perda de carga distribuída; neste tipo de líquido, além de depender da taxa de deformação imposta, dá-se a presença de partículas sólidas consideráveis que impedem uma determinação confiável da viscosidade em viscosímetros e reômetros disponíveis no mercado. Chen \& Hashimoto (1976) determinaram constantes reológicas para águas residuárias da bovinocultura, suinocultura e da avicultura, porém, foram retiradas quase todas as partículas sólidas, de modo a se obter um líquido com baixa concentração de sólidos totais que permitisse sua passagem no reômetro.

Visto que a perda de carga distribuída decorre do atrito entre as diversas camadas do escoamento (efeito da viscosidade) e ainda do atrito entre o líquido e as paredes do conduto (efeito da rugosidade), faz-se oportuno o desenvolvimento de novos métodos para a determinação desse parâmetro, extremamente relevante para dimensionamento de projetos.

Dentre as metodologias consagradas para determinação de perda de carga distribuída na área de projetos de irrigação, destaca-se a equação de Hazen \& Williams (1963), devido à sua simplicidade de uso e fundamentada para o regime turbulento pleno. O trabalho desses autores resultou de um estudo estatístico cuidadoso no qual foram considerados os dados experimentais disponíveis, obtidos anteriormente por um grande número de pesquisadores, além de observações próprias. Segundo os autores, a expressão determinada é teoricamente correta, pois a diferença de seus expoentes é de 3,02 e apresenta uma diferença desprezível sobre o valor teórico. Esses expoentes também foram estabelecidos para resultarem as menores variações do coeficiente numérico $C$ para tubos de mesmo grau de rugosidade; em conseqüência, este coeficiente é, tanto quanto possível e praticável, uma função quase que exclusiva da natureza das paredes.

A grande aceitação que teve a fórmula permitiu que se obtivessem valores bem determinados do coeficiente C, condições em que se pode estimar o envelhecimento dos tubos; trata-se de uma fórmula que pode ser satisfatoriamente aplicada para qualquer tipo de conduto e material, cujos limites de aplicação são os diâmetros de 50 a 3500 mm, que causam pouco entupimento, o que pode tornar tal equação bastante prática para o líquido oriundo de águas residuárias; entretanto, a grande limitação do método dos autores foi desprezar o efeito do líquido em seu modelo matemático, pois trabalhou apenas para água pura. A inclusão dos efeitos do líquido na perda de carga seguindo a equação universal, pode ser feita mediante a viscosidade, porém, o trabalho de Carstens \& Addie (1981) aponta que a concentração de sólidos totais também se constitui em outro parâmetro a ser utilizado na determinação de perda de carga distribuída.

A partir do exposto e se considerando principalmente a dificuldade de determinar respectiva viscosidade e da extrema falta de dados que possibilitem a determinação da perda de carga distribuída da água residuária da suinocultura, objetivou-se com este trabalho encontrar fatores empíricos de correção, para serem aplicados à equação de Hazen \& Williams (1963), considerando-se apenas a concentração de sólidos totais.

\section{MATERIAL E MÉTODOS}

Utilizaram-se, neste trabalho, dados coletados por Sampaio (1999), no qual o autor avaliou as perdas de carga distribuídas nas tubulações de aço zincado, com diâmetros de 73,54, 99,82, 125,24 e 150,42 mm, ferro galvanizado com diâmetros de 53,75, 84,01, 105,90, 130,30 e 155,57 mm, e PVC com diâmetros de 52,61, 79,99, 103,20, 127,31 e 153,43 mm; utilizou-se como líquido circulante a água e a água residuária da suinocultura (ARS) nas concentrações de sólidos totais de 0,08, 0,98, 2,08, 4,73, 7,66 e 8,56\%, que constituíram os líquidos avaliados, em que os respectivos pesos específicos foram de 1005,48, 1012,26, 1019,06, 1026,46 e $1030,04 \mathrm{~kg} \mathrm{~m}^{-3}$. A ARS foi proveniente de águas residuárias de pocilgas, sendo passados por uma peneira feita com tela utilizada para viveiro de pássaros, a fim de se retirar o material grosseiro e outras impurezas indesejáveis (como folhas, pedaços de madeira, entre outros), que poderiam causar o entupimento da tubulação.

No trabalho de Sampaio (1999) o líquido foi bombeado através de moto-bomba até o reservatório superior, de onde escoava, por gravidade, para a tubulação de testes; depois de passar pelos tubos era descarregado no reservatório inferior, no qual era bombeado para o reservatório superior fechando o ciclo.

As tubulações se dispunham em nível, com comprimento útil de $18 \mathrm{~m}$, constituídas de 4 tubos de $6 \mathrm{~m}$ de comprimento e os piezômetros dispostos no meio de cada tubo e assim se formavam trechos de $3 \mathrm{~m}$ de comprimento à jusante e à montante, podendo garantir o escoamento plenamente desenvolvido. 
Tentou-se evitar os erros decorrentes do uso inadequado do aparelho de medição, do observador e também da digestão ocorrida na água residuária. Em cada observação (teste para determinar um par de dados "vazão versus perda de carga”), foram feitas 20 leituras em cada piezômetro além dos 4 piezômetros instalados permitir determinar a linha piezométrica do trecho útil (18 m). Destaca-se que essas leituras foram feitas em tempos diferentes de armazenamento o que provocou uma qualidade química diferente na água residuária, porém não alterou significativamente a concentração de sólidos totais, corroborando com as observações de produtores rurais locais quando citam que as partículas sólidas que passam no trato digestivo do suíno são de difícil tratamento em lagoas de estabilização.

Deste modo, cada observação de perda de carga é uma média de 20 repetições e coeficiente de variação máximo encontrado de $2,11 \%$, ou seja, todos os valores observados foram bastante precisos; também visando contribuir para esta precisão, o diâmetro interno usado nos piezômetros foi de $25 \mathrm{~mm}$, de modo a evitar entupimentos e diminuir as pequenas oscilações ocorridas apenas para as menores vazões.

Coletou-se, em campo, uma série superior a 15 dados de vazão e perda de carga para cada tubulação ensaiada e concentração de sólidos totais, considerando-se apenas as 10 observações, que resultaram no melhor ajuste dos dados, aproximadamente na faixa de regime turbulento $\left(1,49200 \times 10^{6} \leq\right.$ Rey $\left.\leq 24,83679 \times 10^{6}\right)$; assim, os dados constatados como mais susceptíveis às pequenas oscilações observadas por Sampaio (1999) foram descartados. Os valores do coeficiente de rugosidade $C$ das tubulações utilizadas se compunham das médias aritméticas de 10 observações, determinadas a partir dos dados levantados em campo, para água pura, antes e após todos os experimentos, e estimados por meio da equação de Hazen \& Williams (1963) (PVC - $145 \pm 5 \%$; Aço zincado - $164 \pm 3,1 \%$; Ferro galvanizado $-153 \pm 3,7 \%)$.

A vazão foi medida através de um diafragma, com diâmetro de 78,1 mm, situado a aproximadamente $15 \mathrm{~m}$ antes da tubulação avaliada.

De posse dos dados coletados por Sampaio (1999), constituídos pela vazão, perda de carga, coeficientes de rugosidade, diâmetros e concentração de sólidos totais das ARS, os mesmos foram trabalhados usando-se a metodologia descrita a seguir, através da qual se estimaram os fatores de correção para equação de Hazen \& Williams (1963).

Regressões lineares simples foram trabalhadas entre os dados observados de perda de carga contínua para os líquidos avaliados e dados estimados por meio da equação de Hazen \& Wiliams (1963) considerando-se que o líquido escoante fosse água, para estimar os fatores de correção.

Correções foram feitas aos dados observados para ARS em função do peso específico de cada concentração, pois se considerou líquido padrão a água, ou seja, a unidade de perda de carga usada em todas as análises foi metro de coluna de água (mca) e não metro de coluna de água residuária.

Com o paradoxo existente entre a precisão e praticidade do uso de fatores de correção para a equação de Hazen \& Wiliams (1963), avaliaram-se duas metodologias distintas na sua determinação, em que na primeira, denominada fatores de correção parcial, se determinou um fator de correção para cada concentração e tipo de tubulação avaliada, resultando em 15 valores e, na segunda, foram estimados 5 fatores de correção já que são, em função apenas da concentração, denominada fatores de correção global. Em cada metodologia se analisou o total de 1065 pares de dados de perda de carga estimada e observada; de posse desses fatores de correção, determinou-se um modelo matemático que melhor descrevesse o comportamento desses fatores com a concentração.

Para maior facilidade de apresentação e discussão dos resultados é descrita, a seguir, a simbologia das variáveis mais relevantes do trabalho:

$\mathrm{Hf}_{\mathrm{II}-\mathrm{W}}$ - perda de carga sem correção para ARS

$\mathrm{Hf}_{\text {II-Wcorr }}$ - perda de carga corrigida para ARS

$\mathrm{Hf}_{\mathrm{ARS}}$ - perda de carga observada na ARS

\section{RESULTADOS E DISCUSSÃO}

\section{Fatores de correção parciais}

Pode-se observar, na Figura 1, que o aumento da concentração de sólidos totais da ARS proporcionou maior desvio entre a perda de carga calculada pela equação e aquela observada na água residuária. Apesar dos gráficos apresentarem comportamento apenas das concentrações de 0,08 e $8,56 \%$ da tubulação de ferro galvanizado, o mesmo efeito foi notado em todas as concentrações e tipos de tubulação. Na Tabela 1 se apresentam os respectivos coeficientes angulares e de determinação, encontrados para todas as concentrações e tubulações.
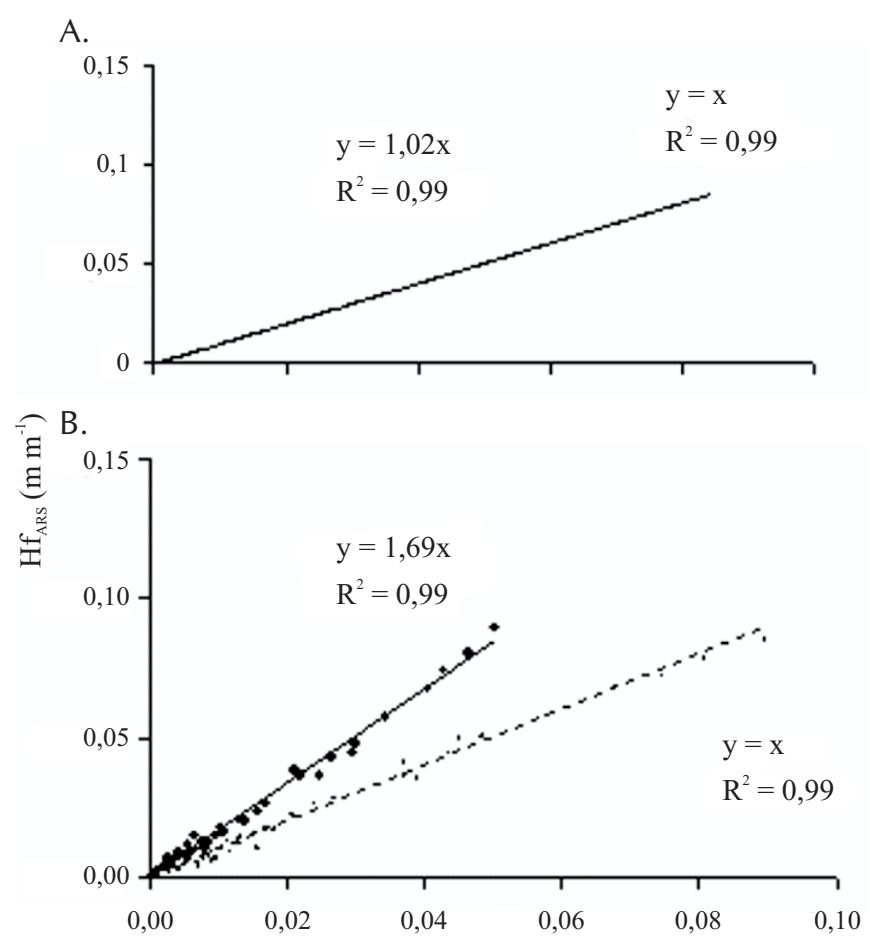

Figura 1. Ajustes entre $\mathrm{Hf}_{\mathrm{H}-\mathrm{W}}$ e $\mathrm{Hf}_{\mathrm{ARS}}(-)$; e $\mathrm{Hf}_{\mathrm{H}-\mathrm{WCorr}}$ e $\mathrm{Hf}_{\mathrm{ARS}}(----)$, nas concentrações de $0,08 \%$ (A) e $8,56 \%$ (B), para tubulação de ferro galvanizado 
Tabela 1. Coeficientes angulares e de determinação encontrados nas correlações realizadas, utilizando-se fatores de correção parcial

\begin{tabular}{|c|c|c|c|c|}
\hline \multirow{2}{*}{$\begin{array}{c}\text { Concentração } \\
\text { ARS (\%) }\end{array}$} & \multicolumn{2}{|c|}{ Coeficientes Angulares } & \multicolumn{2}{|c|}{ Coeficientes de Determinação } \\
\hline & $\mathrm{Hf}_{\mathrm{H}-\mathrm{w}} \times \mathrm{Hf}_{\text {ARS }}$ & $\mathrm{Hf}_{\mathrm{H} \text {-wCorr }} \times \mathrm{Hf}_{\text {ARS }}$ & $\mathrm{Hf}_{\mathrm{H}-\mathrm{w}} \times \mathrm{Hf}_{\mathrm{ARS}}$ & $\mathrm{Hf}_{\mathrm{H} \text {-WCorr }} \times \mathrm{Hf}_{\text {ARS }}$ \\
\hline \multicolumn{5}{|c|}{ Aço Zincado } \\
\hline 0,08 & 0,9556 & 1,00 & 0,9953 & 0,9954 \\
\hline 0,98 & 1,0129 & 1,00 & 0,9972 & 0,9972 \\
\hline 2,08 & 1,1167 & 1,00 & 0,9952 & 0,9954 \\
\hline 4,73 & 1,4177 & 1,00 & 0,9920 & 0,9921 \\
\hline 7,66 & 1,5613 & 1,00 & 0,9934 & 0,9938 \\
\hline 8,56 & 1,6037 & 1,00 & 0,9936 & 0,9939 \\
\hline \multicolumn{5}{|c|}{ Ferro Galvanizado } \\
\hline 0,08 & 1,0177 & 1,00 & 0,9985 & 0,9984 \\
\hline 0,98 & 1,0451 & 1,00 & 0,9992 & 0,9992 \\
\hline 2,08 & 1,1 & 1,00 & 0,9981 & 0,9982 \\
\hline 4,73 & 1,2448 & 1,00 & 0,9944 & 0,9946 \\
\hline 7,66 & 1,5729 & 1,00 & 0,9948 & 0,9949 \\
\hline 8,56 & 1,6939 & 1,00 & 0,9919 & 0,9919 \\
\hline \multicolumn{5}{|c|}{ PVC } \\
\hline 0,08 & 0,9593 & 1,00 & 0,9963 & 0,9964 \\
\hline 0,98 & 0,9914 & 1,00 & 0,9993 & 0,9993 \\
\hline 2,08 & 1,0386 & 1,00 & 0,9972 & 0,9973 \\
\hline 4,73 & 1,1383 & 1,00 & 0,9977 & 0,9978 \\
\hline 7,66 & 1,3042 & 1,00 & 0,9871 & 0,9884 \\
\hline 8,56 & 1,3962 & 1,00 & 0,9863 & 0,9873 \\
\hline
\end{tabular}

Em razão de apresentarem os maiores desvios, as concentrações mais elevadas também são aquelas que mostraram maiores fatores de correção, conforme a Tabela 2; percebese, ainda, que os fatores de correção foram superiores nas tubulações de ferro galvanizado e aço zincado e, por último, na tubulação de PVC.

Tabela 2. Fatores de correção parcial para equação de Hazen \& Williams (1963), em função da concentração de ARS, para as tubulações de aço zincado, ferro galvanizado e PVC

\begin{tabular}{lcccccc}
\hline \multirow{3}{*}{ Tubulação } & \multicolumn{6}{c}{ Concentração da ARS (\%) } \\
\cline { 2 - 7 } & $\mathbf{0 , 0 8}$ & $\mathbf{0 , 9 8}$ & $\mathbf{2 , 0 8}$ & $\mathbf{4 , 7 3}$ & $\mathbf{7 , 6 6}$ & $\mathbf{8 , 5 6}$ \\
\cline { 2 - 7 } & \multicolumn{5}{c}{ Fatores de correção parcial - FP } \\
Aço zincado & 0,96 & 1,02 & 1,12 & 1,42 & 1,57 & 1,64 \\
Ferro galvanizado & 1,02 & 1,05 & 1,10 & 1,25 & 1,58 & 1,70 \\
PVC & 0,96 & 0,99 & 1,04 & 1,14 & 1,31 & 1,41 \\
Média & 0,98 & 1,02 & 1,09 & 1,27 & 1,49 & 1,58 \\
\hline
\end{tabular}

Com a finalidade de generalizar os fatores de correção parcial (Fp) encontrados para cada tipo de tubulação, determinou-se um modelo matemático que melhor represente seu comportamento em função da concentração estudada, para as tubulações de aço zincado, ferro galvanizado e PVC, como se observa nas Eq. 1, 2 e 3, respectivamente e na Figura 2. Na tentativa de simplificar ainda mais os fatores de correção, quando calculada a perda de carga distribuída da ARS, estimou-se uma equação média (Eq. 4), também encontrada na Figura 3. A equação média foi obtida em função da média aritmética entre os fatores de correção parciais encontrados para três tubulações avaliadas na respectiva concentração.

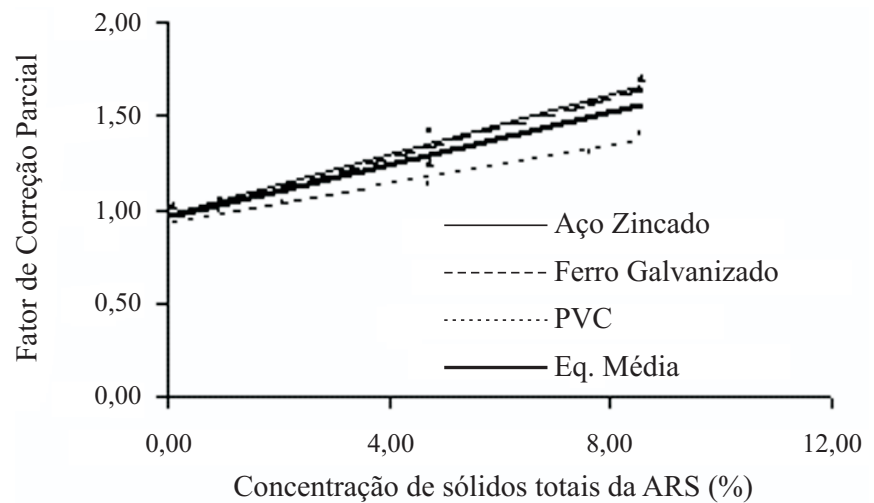

\begin{tabular}{lll} 
Aço & $\mathrm{Fp}=0,0805$. Conc $+0,9678$ & $\mathrm{R}^{2}=0,9834$ \\
Ferro & $\mathrm{Fp}=0,0804$. Conc $+0,9590$ & $\mathrm{R}^{2}=0,9652$ \\
PVC & $\mathrm{Fp}=0,0505$. Conc $+0,9387$ & $\mathrm{R}^{2}=0,9805$ \\
Média & $\mathrm{Fp}=0,0705$. Conc $+0,9551$ & $\mathrm{R}^{2}=0,9952$ \\
em que: & \\
\multicolumn{3}{l}{ Fp - Fator de correção parcial, adimensional } \\
Conc - Concentração de sólidos totais da ARS, $\%$
\end{tabular}

Figura 2. Fatores de correção parcial em função da concentração da ARS

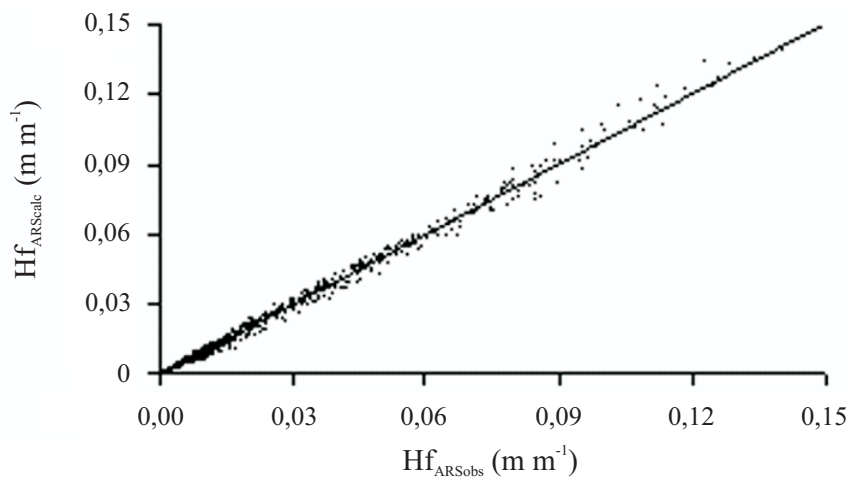

Figura 3. Ajuste entre a perda de carga observada e calculada, usando-se equações parciais e equação média de fatores de correção na equação de Hazen \& Wiliams (1963)

\section{Fatores de correção global}

Nesta determinação se utilizam pares de dados correspondentes a cada concentração e tipo de tubulação; deste modo, todas as análises realizadas para a metodologia anterior foram também desenvolvidas, entretanto, como os dados foram agrupados em função apenas da concentração, os resultados obtidos foram mais resumidos.

As correlações entre as perdas de carga determinadas para a água e observadas para ARS e a correlação entre as perdas de carga observada para ARS e determinada a partir do fator de correção específico para cada concentração, são mostradas na Figura 4. Os valores dos coeficientes angulares e de determinação podem ser vistos na Tabela 3.

Observa-se que, como no caso anterior, o desvio encontrado foi maior para as concentrações mais elevadas, corroborando ainda mais com o trabalho de Sampaio (1999), de que a perda de carga aumentou na medida em que se aumentou a concentração da ARS.

Assim, os fatores de correção global também foram maiores para as concentrações mais elevadas, conforme a Tabela 4 


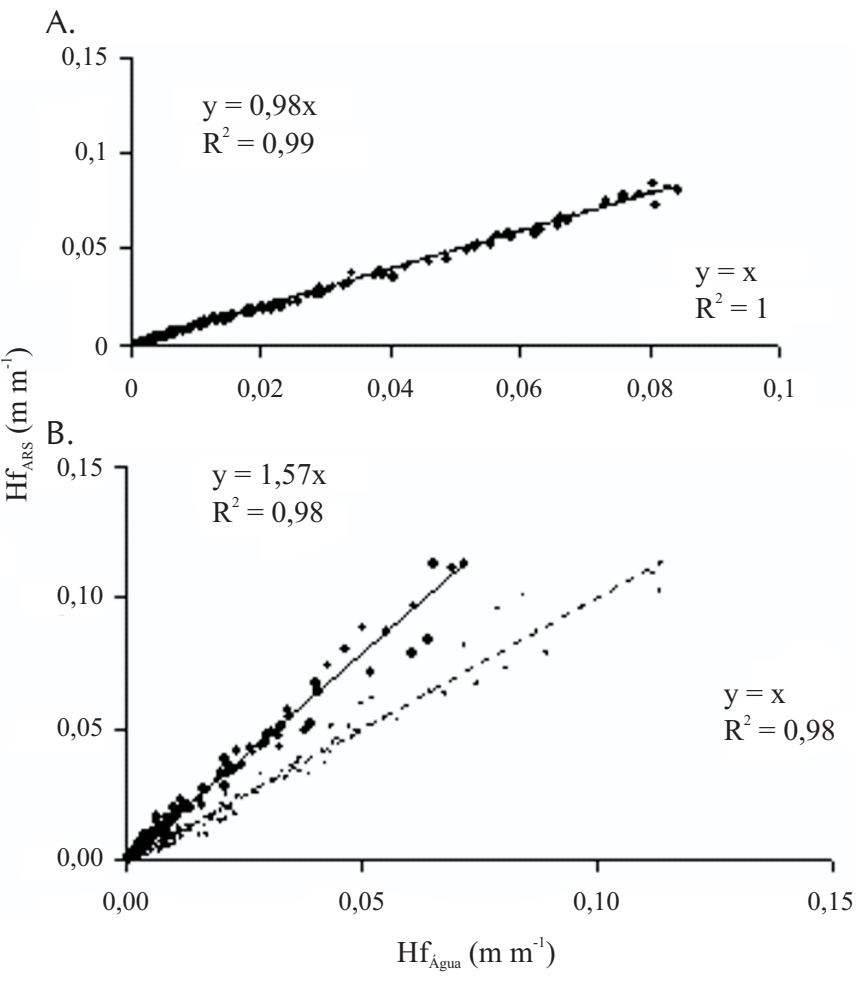

Figura 4. Ajuste entre $\mathrm{Hf}_{\mathrm{H}-\mathrm{W}}$ e $\mathrm{Hf}_{\mathrm{ARS}}(-)$ ) e $\mathrm{Hf}_{\mathrm{H}-\mathrm{WCorr}}$ e $\mathrm{Hf}_{\mathrm{ARS}}(----)$, nas concentrações de $0,08(4 A)$ e $8,56 \%$ (4B)

Tabela 3. Coeficientes angulares e de determinação encontrados nas correlações realizadas, utilizando-se fatores de correção global

\begin{tabular}{|c|c|c|c|c|}
\hline \multirow{2}{*}{$\begin{array}{c}\text { Concentração } \\
\text { ARS (\%) }\end{array}$} & \multicolumn{2}{|c|}{ Coeficientes Angulares } & \multicolumn{2}{|c|}{ Coeficientes de Determinação } \\
\hline & $\mathrm{Hf}_{\mathrm{H}-\mathrm{W}} \times \mathrm{Hf}_{\text {ARS }}$ & $\mathrm{Hf}_{\mathrm{H}-\mathrm{WCorr}} \times \mathrm{Hf}_{\mathrm{ARS}}$ & $\mathrm{Hf}_{\mathrm{H}-\mathrm{W}} \times \mathrm{Hf}_{\mathrm{ARS}}$ & $\mathrm{Hf}_{\mathrm{H}-\mathrm{WCor}} \times \mathrm{Hf}_{\mathrm{ARS}}$ \\
\hline 0,08 & 0,9858 & 1,0000 & 0,9956 & 1,000 \\
\hline 0,98 & 1,0087 & 1,0000 & 0,9985 & 0,9982 \\
\hline 2,08 & 1,0725 & 1,0000 & 0,9954 & 0,9955 \\
\hline 4,73 & 1,2169 & 1,0000 & 0,9833 & 0,9841 \\
\hline 7,66 & 1,4664 & 1,0000 & 0,9763 & 0,9776 \\
\hline 8,56 & 1,5680 & 1,0000 & 0,9800 & 0,9808 \\
\hline
\end{tabular}

Tabela 4. Fatores de correção global para a equação de Hazen \& Williams (1963), em função da concentração de ARS

\begin{tabular}{cc}
\hline Concentração ARS $(\%)$ & Fatores de correção global $\left(\mathbf{F}_{\mathbf{g}}\right)$ \\
0,08 & 1,0 \\
0,98 & 1,0091 \\
2,08 & 1,0751 \\
4,73 & 1,2273 \\
7,66 & 1,4830 \\
8,56 & 1,5847 \\
\hline
\end{tabular}

seguindo, ainda, as afirmações de Kumar et al. (1972), Bashford et al. (1977) e Steffe \& Morgan (1986), em que na maior parte dos casos o aumento da concentração de sólidos totais tende a aumentar a turbulência no líquido e, conseqüentemente, a perda de carga ocorrida no trecho. A partir dos valores da Tabela 4 buscou-se o modelo matemático (Eq. 5) que melhor descrevesse este comportamento.

$$
\mathrm{F}_{\mathrm{g}}=0,0701 . \text { Conc }+0,95 \quad \mathrm{R}^{2}=0,98
$$

em que:

$$
\begin{gathered}
\mathrm{F}_{\mathrm{g}} \text { - Fator de correção global, adimensional } \\
\text { Conc - Concentração de sólidos totais da ARS, \% }
\end{gathered}
$$

Notam-se, ainda na Tabela 2, fatores de correção com valores inferiores a 1,0, principalmente nas menores concentrações, fato que pode ser atribuído, sem dúvida, à metodologia empregada, pois na Tabela 4 em que se usou outra metodologia o valor é igual a 1,0.

Percebe-se, desta forma, que os fatores de correção chegaram a apresentar valores da ordem de 1,70 (Tabela 2) e 1,58 (Tabela 4) contrapondo, portanto, a afirmação de Kumar et al. (1972) em que, independente do tipo de água residuária e de sua concentração, bastaria acrescer-se $10 \%$ no valor da perda de carga.

Apresentam-se, na Figura 5, os pontos observados e estimados de perda de carga, quando se utilizaram os fatores de correção global.

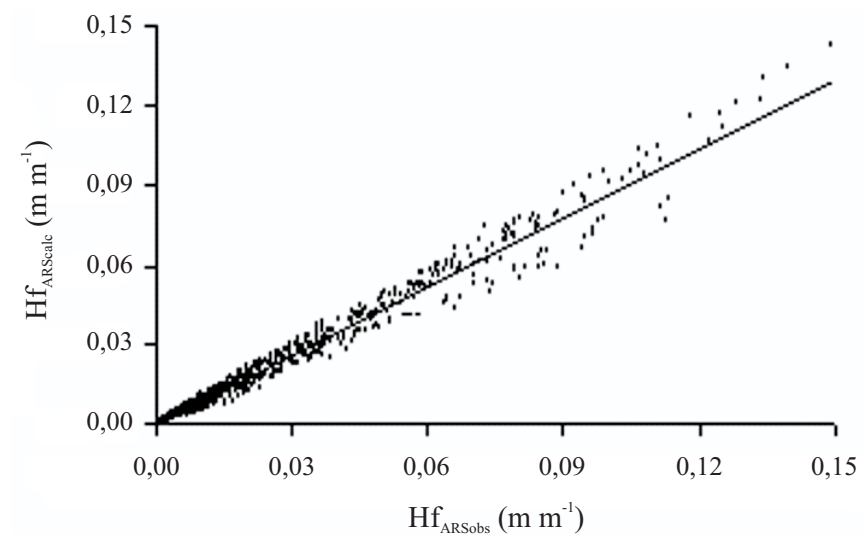

Figura 5. Ajuste entre a perda de carga observada e calculada, usando-se equação de fatores de correção global na equação de Hazen \& Wiliams (1963)

Constatou-se, nas duas metodologias, que a adição de esterco suíno proporcionou aumento da perda de carga, corroborando com as pesquisas de Herman e Stepanoff, citados por Kumar et al. (1972), Bashford et al. (1977) e de Broadfoot et al. (1994). Esses autores justificam o aumento da perda de carga em função do aumento da rugosidade da tubulação, devido à adesão de partículas junto às paredes, porém no experimento de Sampaio (1999) este fato não ocorreu para água residuária da suinocultura.

Percebe-se facilmente que a não consideração dos fatores de correção pode levar a um subdimensionamento hidráulico da ordem de 70\%, observada na maior concentração para metodologia de fatores globais.

\section{CONCLUSÕES}

1. A perda de carga possui comportamento linear em função da concentração de sólidos totais da água residuária, proveniente da suinocultura. 
2. As perdas de carga determinadas a partir dos fatores de correção parcial e global, apresentaram superestimativa e subestimativa média de aproximadamente 0,7 e $13 \%$, respectivamente.

3. Recomenda-se adotar, em projetos hidráulicos para ARS, os fatores de correção parcial, por apresentarem maior precisão.

\section{LITERATURA CITADA}

Bashford, L. L.; Gilbertson, C. B.; Nienaber, J. A.; Tietz, D. Eeffects of ration roughage content on viscosity and theoretical head losses in pipe flow for beef cattle slurry. Transactions of the ASAE, St. Joseph, v.20, n.6, p.1106-1109, 1977.

Broadfoot, R.; Viana, M. J. de; Daniel, J. E.; Morris, J. T.; De Viana, M. J.; Egan, B. T. Pressure losses in pipe fittings at low Reynolds number. In: Conference of the Australian Society of Sugar Cane Technologists held at Townsville,
1994, Queensland. Proceedings... Queensland: Sugar Research Institute, 1994, p.330-339.

Carstens M. R.; Addie G. A sand-water slurry experiment. Journal of the Hidraulics Division, New York, v.107, n.4, p.501-507, 1981.

Chen, Y. R.; Hashimoto, A. G. Rheological properties of aerated poultry waste slurries. Transactions of the ASAE, St. Joseph, v.19, n.5, p.128-133, 1976.

Hazen, A.; Williams, G. S. Hydraulic tables. New York: John Wiley \& Sons, 1963. 120p.

Kumar, M.; Bartelett, H. D.; Mohsenin, N. N. Flow properties of animal waste slurries. Transactions of the ASAE, St. Joseph, v.30, n.2, p.718-722, 1972.

Sampaio, S. C. Perda de carga em tubulações comerciais conduzindo águas residuárias de bovinocultura e suinocultura. Viçosa: UFV, 1999. 158p. Tese Doutorado

Steffe, J. F.; Morgan, R. G. Pipeline design and pump selection for non-newtonian fluid foods. Food Technology, Chicago, v.40, n.12, p.78-85, 1986. 